SINP/TNP/04-04, hep-th/0403083

\title{
Supersymmetric quantum mechanics on noncommutative space
}

\author{
Pijush K. Ghosh* \\ Theory Division, \\ Saha Institute of Nuclear Physics, \\ Kolkata 700 064, India.
}

\begin{abstract}
We construct supersymmetric quantum mechanics in terms of two real supercharges on noncommutative space in arbitrary dimensions. We obtain the exact eigenspectra of the two and three dimensional noncommutative superoscillators. We further show that a reduction in the phase-space occurs for a critical surface in the space of parameters. At this critical surface, the energy-spectrum of the bosonic sector is infinitely degenerate, while the degeneracy in the spectrum of the fermionic sector gets enhanced by a factor of two for each pair of reduced canonical coordinates. For the two dimensional noncommutative 'inverted superoscillator', we find exact eigenspectra with a welldefined groundstate for certain regions in the parameter space, which have no smooth limit to the ordinary commutative space.
\end{abstract}

*Electronic address: pijush@theory.saha.ernet.in 


\section{INTRODUCTION}

The noncommutative space or the associated algebra arises in many branches of physics

and mathematics 1 ]. The reason behind the recent upsurge in the study of field theory on the noncommutative space is that such theories naturally appear in a particular lowenergy limit of open string theory on D-brane backgrounds in the presence of a constant antisymmetric tensor field|2]. There are already many interesting results on different aspects of both perturbative [3] and non-perturbative[4] non-commutative field theory [5], 6, 6]. The study of supersymmetric field theories on noncommutative spaces with the standard anticommuting [8] or non-anticommuting [9, 10] odd coordinates is also an active area of research.

Along the same line of development, quantum mechanics on the noncommutative space has been studied extensively [11, 12, 13, 14, 15, 16, 17, 18, 19, 20]. The complete eigenstates of the noncommutative oscillator [14, 15, 18, 19] have been found analytically in two and higher dimensions. In particular, the spectrum of the noncommutative oscillator is shown to be identical to that of an anisotropic oscillator on the corresponding commutative space. The frequencies of the anisotropic oscillator are, in general, a function of the parameters appearing in the noncommutative algebra of the phase-space operators. In the appropriate commutative limit, the spectrum of an isotropic oscillator on commutative space is recovered. No other nontrivial quantum mechanical problem is known to be completely solvable on the noncommutative plane or higher dimensions.

Supersymmetric quantum mechanics on commutative space is a well developed subject 21]. The study of supersymmetric quantum mechanics enriched us in understanding many subtle issues of supersymmetric field theory in a much simpler way. Further, different ideas and techniques emerging from this subject can be used to solve algebraically a host of quantum mechanical problems arising in diverse branches of physics, including those found in any standard textbook on quantum mechanics. The $\star$-product formulation of supersymmetric quantum mechanics on commutative space is also known [22]. 
It is natural to enquire at this juncture about the present status of the supersymmetric quantum mechanics on the noncommutative space. Recently, the Pauli equation on the noncommutative plane was shown to be supersymmetric with the gyro-magnetic ratio $g=2$ [23]. The superoscillator on the noncommutative plane has also been constructed[24]. However, we find that a general and uniform formulation of the $\mathcal{N}=2$ supersymmetric quantum mechanics on noncommutative space is lacking. For example, the supersymmetric constructions in Refs. [23, 24] are not suitable, (i) for dimensions $N \geq 3$, (ii) for describing quantum mechanical Hamiltonian with any arbitrary superpotential and (iii) for accommodating more general noncommutativities among phase-space operators.

The purpose of this paper is to present a formulation of supersymmetric quantum mechanics on noncommutative space without the shortcomings discussed above. In particular, we construct supersymmetric quantum mechanics in terms of $\mathcal{N}=2$ real supercharges on noncommutative space in arbitrary dimensions. This construction is valid for any arbitrary superpotential and accommodates a very large class of noncommutativities among phasespace operators. We study the superoscillator on two and three dimensional noncommutative spaces in some detail.

We obtain the complete spectrum of the two dimensional superoscillator analytically. The spectrum is identical to that of an anisotropic superoscillator on the commutative plane. The frequencies of the anisotropic superoscillators are functions of the parameters appearing in the non-commutative algebra of the phase-space operators. In the commutative limit, the isotropic superoscillator is recovered. Further, we determine the spectrum of the 'inverted superoscillator' on the noncommutative plane with a well defined groundstate for certain regions in the parameter space. There is no smooth limit to the commutative space from these regions of the parameter space. Although, the Hamiltonian of the 'inverted superoscillator' on the noncommutative plane is non-hermitian and not semi-positive definite, the spectrum obtained is indeed real and positive.

A general analysis of the three dimensional noncommutative superoscillator is messy and complicated. So, we present the energy spectrum for a few specific choices of the parameters. 
For all these cases, the energy spectrum is identical to that of an anisotropic superoscillator on three dimensional commutative space. The commutative limit for all these cases is smooth and reproduces the well known results for usual isotropic superoscillator in three dimensions.

We show that for both two and three dimensional noncommutative superoscillators, a reduction in the phase space occurs for a critical surface in the parameter space. Consequently, the energy spectrum in the bosonic sector becomes infinitely degenerate, while the degeneracy in the fermionic sector gets enhanced by a factor of two for each pair of reduced canonical coordinates. The disparity in the enhancement of degeneracy between the bosonic and the fermionic sector can be attributed to the fact that bosonic quantum number can take any non-negative integer values, while a fermionic quantum number can take only two values.

The plan of the paper is the following. We introduce the noncommutative algebra among different phase-space operators in the next section. We also construct $\mathcal{N}=2$ supersymmetric quantum mechanics on the noncommutative space for an arbitrary superpotential. The super-Hamiltonian for the particular case of superoscillator in $N$ dimensions is obtained. In Sec. III, we study the two dimensional superoscillator in detail and obtain its energy spectrum. Different limiting cases including the 'inverted superoscillator' is discussed in this section. The superoscillator in three dimensions is discussed in Sec. IV. Finally, we conclude in Sec. V. In Appendix A, the procedure for diagonalizing the three dimensional bosonic Hamiltonian is discussed. In Appendix B, the matrix representation of the fermionic Hamiltonian for $N=3$ is given.

\section{GENERAL FORMULATION}

Consider the noncommutative algebra,

$$
\left[\hat{x}_{i}, \hat{x}_{j}\right]=i \theta_{i j}, \quad\left[\hat{p}_{i}, \hat{p}_{j}\right]=i B_{i j}, \quad\left[\hat{x}_{i}, \hat{p}_{j}\right]=i \delta_{i j}+i\left(1-\delta_{i j}\right) C_{i j}, \quad i, j=1,2, \ldots, N,
$$

where $\theta_{i j}$ and $B_{i j}$ are real, antisymmetric matrices and are independent of the hermitian operators $\hat{x}_{i}, \hat{p}_{i}$. The diagonal elements of the matrix $C_{i j}$ are taken to be zero and its off- 
diagonal elements do not depend on the space and momentum coordinates. We introduce $2 N$ elements $\xi_{i}$ satisfying the following real Clifford algebra,

$$
\left\{\xi_{i}, \xi_{j}\right\}=2 g_{i j}, \quad g_{i j}=\left(\begin{array}{cc}
-\mathcal{I} & 0 \\
0 & \mathcal{I}
\end{array}\right),
$$

where $\mathcal{I}$ is an $N \times N$ identity matrix. The signature of the metric $g_{i j}$ is such that the square of the $\xi_{i}$ 's is equal to 1 or -1 depending on whether $i>N$ or $i \leq N$, respectively. In particular,

$$
\xi_{N+i}^{2}=-\xi_{i}^{2}=1, \quad i=1,2, \ldots N
$$

In this paper, we use a particular matrix representation of the Clifford algebra so that the following additional relations are also satisfied,

$$
\xi_{i}^{\dagger}=-\xi_{i}, \quad \xi_{N+i}^{\dagger}=\xi_{N+i}
$$

where $X^{\dagger}$ denotes the hermitian adjoint of $X$. Such a matrix representation of the Clifford algebra is required to construct hermitian Hamiltonian within our approach. We further introduce the hermitian operator $\gamma_{5}$,

$$
\gamma_{5}=\xi_{1} \xi_{2} \ldots \xi_{2 N-1} \xi_{2 N}
$$

which anticommutes with all the $\xi_{i}$ 's and $\gamma_{5}^{2}=1$. The elements of the Clifford algebra $\xi_{i}, \xi_{N+i}$ commute with the noncommutative bosonic variables $\hat{x}_{i}$ and $\hat{p}_{i}$,

$$
\left[\hat{x}_{i}, \xi_{j}\right]=\left[\hat{p}_{i}, \xi_{j}\right]=0, \quad\left[\hat{x}_{i}, \xi_{N+j}\right]=\left[\hat{p}_{i}, \xi_{N+j}\right]=0, \quad \forall i, j
$$

It naturally follows that $\gamma_{5}$ also commutes with all the bosonic coordinates.

We now introduce the supercharges $Q_{1}$ and $Q_{2}[25,26]$,

$$
Q_{1}=\frac{1}{\sqrt{2}} \sum_{i=1}^{N}\left[-i \xi_{i} \hat{p}_{i}+\xi_{N+i} \hat{W}_{i}\right], \quad Q_{2}=-i \gamma_{5} Q_{1} .
$$

The superpotential $\hat{W}_{i}$ are real functions of the noncommutative coordinate $\hat{x}_{i}$ and in general $\left[\hat{W}_{i}, \hat{W}_{j}\right] \neq 0$. Note that both $Q_{1}$ and $Q_{2}$ are constructed to be hermitian operators for real $\hat{W}_{i}$. The supercharges $Q_{1}$ and $Q_{2}$ satisfy the following standard superalgebra,

$$
\left\{Q_{\alpha}, Q_{\beta}\right\}=2 \delta_{\alpha, \beta} H, \quad\left[H, Q_{\alpha}\right]=0, \quad \alpha, \beta=1,2
$$


where the Hamiltonian $H$ is given by,

$$
H=\frac{1}{2} \sum_{i=1}^{N}\left(\hat{p}_{i}^{2}+\hat{W}_{i}^{2}\right)-\frac{i}{4} \sum_{i, j=1}^{N}\left(B_{i j} \xi_{i} \xi_{j}+2 \xi_{i} \xi_{N+j}\left[\hat{p}_{i}, \hat{W}_{j}\right]+i \xi_{N+i} \xi_{N+j}\left[\hat{W}_{i}, \hat{W}_{j}\right]\right) .
$$

The Hamiltonian $H$ is hermitian, since it is given by the square of the hermitian operator $Q_{1}\left(Q_{2}\right)$. The hermiticity of $H$ can also be checked explicitly using the equation (44). The term containing $B_{i j}$ arises due to the noncommutativity among momentum operators. Similarly, the last term in $H$ that is proportional to $\left[\hat{W}_{i}, \hat{W}_{j}\right]$ arises due to the noncommutativity among space coordinates. Such a term is absent for supersymmetric quantum mechanics on commutative space.

A few comments are in order at this point.

(i) If we allow $\theta_{i j}, B_{i j}$ and $C_{i j}$ to be functions of the noncommutative coordinates $\hat{x}_{i}, \hat{p}_{i}$ instead of c-number matrices, the whole analysis up to the construction of the superHamiltonian (9) remains valid. It is worth mentioning here that the Jacobi identities severely restrict the choice of the operators $\theta_{i j}, B_{i j}, C_{i j}$ for such more general theories.

(ii) In the standard construction of supersymmetric quantum mechanics on the commutative space, one usually introduces fermionic variables $\psi_{i}$ and its conjugate $\psi_{i}^{\dagger}$,

$$
\psi_{i}=\frac{i}{2}\left(\xi_{i}-\xi_{N+i}\right), \quad \psi_{i}^{\dagger}=\frac{i}{2}\left(\xi_{i}+\xi_{N+i}\right)
$$

so that the eigenstates can be labeled in terms of the total fermion number $N_{F}=\sum_{i} \psi_{i}^{\dagger} \psi_{i}$. However, one can check that $H$ contains term of the form $\psi_{i} \psi_{j}, \psi_{i}^{\dagger} \psi_{j}^{\dagger}$ for $B_{i j}, \theta_{i j} \neq 0$, implying $N_{f}$ is not a conserved quantity.

(iii) Let us define another set of supercharges $q_{1}$ and $q_{2}$,

$$
q_{1}=-\frac{1}{\sqrt{2}} \sum_{i=1}^{N}\left(\xi_{N+i} \hat{p}_{i}+i \xi_{i} \hat{W}_{i}\right), \quad q_{2}=-i \gamma_{5} q_{1}
$$

These two supercharges satisfy the standard superalgebra (8) with the Hamiltonian $h \equiv$ $q_{1}^{2}=q_{2}^{2}$,

$$
h=\frac{1}{2} \sum_{i=1}^{N}\left(\hat{p}_{i}^{2}+\hat{W}_{i}^{2}\right)+\frac{i}{4} \sum_{i, j=1}^{N}\left(B_{i j} \xi_{N+i} \xi_{N+j}-2 \xi_{i} \xi_{N+j}\left[\hat{p}_{j}, \hat{W}_{i}\right]+i \xi_{i} \xi_{j}\left[\hat{W}_{i}, \hat{W}_{j}\right]\right) .
$$


In the commutative limit $B_{i j}=\theta_{i j}=C_{i j}=0$, the two Hamiltonian $H$ and $h$ are identical. Moreover, in the same limit, the pair of charges $\left(Q_{1}, q_{1}\right)$ and the pair $\left(Q_{2}, q_{2}\right)$ satisfy the superalgebra (8) separately. Although, $\left(Q_{1}, Q_{2}, q_{1}, q_{2}\right)$ do not close under an enlarged $\mathcal{N}=4$ superalgebra, we have the freedom of choosing any pair of the supercharges $\left\{\left(Q_{1}, Q_{2}\right),\left(q_{1}, q_{2}\right),\left(Q_{1}, q_{1}\right),\left(Q_{2}, q_{2}\right)\right\}$ for a given $\mathcal{N}=2$ super-Hamiltonian $H=h$ on the commutative space. However, if we take any of the parameters $\theta_{i j}, B_{i j}, C_{i j}$ non-zero, such a freedom is completely lost. We are compelled to choose either the pair $\left(Q_{1}, Q_{2}\right)$ or $\left(q_{1}, q_{2}\right)$ and of-course, in general, the super-Hamiltonian $H$ and $h$ are not identical.

\section{A. Superoscillators}

We will be working with $Q_{1}, Q_{2}$ and $H$ in the rest of the paper. We also consider $\theta_{i j}, B_{i j}, C_{i j}$ as constant matrices from now onward. For the case of superoscillator, we choose,

$$
\hat{W}_{i}=\omega \hat{x}_{i}
$$

The Hamiltonian now reads,

$$
\begin{aligned}
H & =H_{b}-H_{f} \\
H_{b} & \equiv \frac{1}{2} \sum_{i=1}^{N}\left(\hat{p}_{i}^{2}+\omega^{2} \hat{x}_{i}^{2}\right) \\
H_{f} & \equiv \frac{\omega}{2}\left(\sum_{i=1}^{N} \xi_{i} \xi_{N+i}-\sum_{i \neq j} \xi_{N+i} \xi_{j} C_{i j}\right)+\frac{i}{4} \sum_{i, j=1}^{N}\left(B_{i j} \xi_{i} \xi_{j}-\omega^{2} \theta_{i j} \xi_{N+i} \xi_{N+j}\right) .
\end{aligned}
$$

In the limit of $B_{i j}, \theta_{i j}, C_{i j} \rightarrow 0$, the Hamiltonian of the superoscillator on commutative space is recovered. Note that $H_{b}$ is a function of the noncommutative co-ordinates and the momenta only, whereas $H_{f}$ is solely expressed in terms of the elements of the Clifford algebra. This implies that $H_{b}$ and $H_{f}$ can be diagonalized separately.

A comment is in order at this point. The $N$ dimensional superoscillator is described in terms of $2 N$ elements of the Clifford algebra (2). So, the Hamiltonian $H_{f}$ can be expressed in terms of the linear combination of the $N(2 N-1)$ generators $\Sigma_{i j}^{1,2,3}$,

$$
\Sigma_{i j}^{1}=\frac{i}{4}\left[\xi_{i}, \xi_{j}\right], \quad \Sigma_{i j}^{2}=\frac{i}{4}\left[\xi_{N+i}, \xi_{N+j}\right], \quad \Sigma_{i j}^{3}=\frac{i}{4}\left[\xi_{i}, \xi_{N+j}\right],
$$


of the group $S O(N, N)$ of rank $N$. Thus, in general, the eigenvalues of $H_{f}$ can be expressed in terms of $N$ quantum numbers. Further, in the matrix representation of the Clifford algebra (22), both the generators $\Sigma_{i j}^{1,2,3}$ and the Hamiltonian $H_{f}$ can be expressed in terms of $2^{N} \times 2^{N}$ dimensional matrices. Thus, each of the $N$ quantum numbers can take only two values.

\section{TWO DIMENSIONAL SUPEROSCILLATOR}

We now specialize in this section to the noncommutative plane for which the antisymmetric matrices $B_{i j}$ and $\theta_{i j}$ can be parametrized in terms of single parameters $B$ and $\theta$, respectively. In particular,

$$
C_{12} \equiv \phi_{1}, \quad C_{21} \equiv-\phi_{2}, \quad B_{i j} \equiv \epsilon_{i j} B, \quad \theta_{i j} \equiv \epsilon_{i j} \theta, \quad i, j=1,2 .
$$

With this choice for $B_{i j}$ and $\theta_{i j}$, there are many physically equivalent representations [14, 19] of the algebra (11) in terms of commutative canonically conjugate variables $x_{i}$ and $p_{i}$ satisfying

$$
\left[x_{i}, x_{j}\right]=0,\left[p_{i}, p_{j}\right]=0,\left[x_{i}, p_{j}\right]=i \delta_{i j} .
$$

As shown in Ref. 14, 15, 19], the Hamiltonian $H_{b}$ with $N=2$ can be equivalently written as a two-dimensional anisotropic oscillator. In particular,

$$
\begin{aligned}
H_{b} & =\frac{1}{2}\left[\Omega_{+}\left(p_{1}^{2}+x_{1}^{2}\right)+\Omega_{-}\left(p_{2}^{2}+x_{2}^{2}\right)\right], \\
2 \Omega_{ \pm} & =\sqrt{\left(\omega^{2} \theta-B\right)^{2}+4 \omega^{2}+\omega^{2}\left(\phi_{1}+\phi_{2}\right)^{2}} \pm \sqrt{\left(\omega^{2} \theta+B\right)^{2}+\omega^{2}\left(\phi_{1}-\phi_{2}\right)^{2}}, \quad \kappa>0, \\
2 \Omega_{ \pm} & = \pm \sqrt{\left(\omega^{2} \theta-B\right)^{2}+4 \omega^{2}+\omega^{2}\left(\phi_{1}+\phi_{2}\right)^{2}}+\sqrt{\left(\omega^{2} \theta+B\right)^{2}+\omega^{2}\left(\phi_{1}-\phi_{2}\right)^{2}}, \kappa<0, \\
\Omega_{+} & =\sqrt{\left(\omega^{2} \theta+B\right)^{2}+\omega^{2}\left(\phi_{1}-\phi_{2}\right)^{2}}, \quad \Omega_{-}=0, \kappa=0,
\end{aligned}
$$

where the positive and the negative values of the parameter $\kappa \equiv \omega^{2}\left(1-B \theta+\phi_{1} \phi_{2}\right)$ correspond to two different phases of the noncommutative oscillators, the critical value being $\kappa=0$. The energy eigenvalues $E_{b}$ of $H_{b}$ is,

$$
E_{b}=\left(n_{+}^{b}+\frac{1}{2}\right) \Omega_{+}+\left(n_{-}^{b}+\frac{1}{2}\right) \Omega_{-}
$$


where the quantum numbers $n_{ \pm}^{b}$ can take any non-negative integer values.

In order to diagonalize $H_{f}$, we use the following matrix representation of the Clifford algebra,

$$
\xi_{1}=i \sigma_{1} \otimes \sigma_{2}, \quad \xi_{2}=i \sigma_{2} \otimes \sigma_{2}, \quad \xi_{3}=-\sigma_{3} \otimes \sigma_{2}, \quad \xi_{4}=I \otimes \sigma_{3},
$$

where $\sigma_{1,2,3}$ are the three Pauli matrices and $I$ is a $2 \times 2$ identity matrix. The Hamiltonian $H_{f}$ is a $4 \times 4$ matrix,

$$
H_{f}=\left(\begin{array}{cc}
\alpha & \beta \\
\beta & \alpha
\end{array}\right), \alpha \equiv\left(\begin{array}{cc}
\frac{B}{2} & \frac{\omega}{2}\left(i+\phi_{1}\right) \\
\frac{\omega}{2}\left(-i+\phi_{1}\right) & -\frac{B}{2}
\end{array}\right), \beta \equiv\left(\begin{array}{cc}
-\frac{\omega^{2} \theta}{2} & \frac{\omega}{2}\left(i+\phi_{2}\right) \\
\frac{\omega}{2}\left(-i+\phi_{2}\right) & \frac{\omega^{2} \theta}{2}
\end{array}\right) .
$$

The eigenvalues of $H_{f}$ are,

$$
\left\{-\frac{1}{2}\left(\Omega_{+}-\Omega_{-}\right), \frac{1}{2}\left(\Omega_{+}-\Omega_{-}\right),-\frac{1}{2}\left(\Omega_{+}+\Omega_{-}\right), \frac{1}{2}\left(\Omega_{+}+\Omega_{-}\right)\right\} .
$$

For $\Omega_{-}=0$, there are only two independent eigenvalues $\pm \frac{1}{2} \Omega_{+}$, each of them being doubly degenerate. The frequency $\Omega_{-}$vanishes only in the critical phase $\kappa=0$. Separate discussions are needed for the critical and the non-critical phases.

\section{A. Critical phase $\kappa=0$}

The frequency $\Omega_{-}=0$ in the critical phase. The eigenvalues $E_{f}$ of $H_{f}$ can be written in a closed form as,

$$
-E_{f}=\frac{1}{2} n_{+}^{f} n_{-}^{f} \Omega_{+}, \quad n_{ \pm}^{f}=-1,1
$$

The nonlinear dependence on the 'fermionic' quantum numbers $n_{ \pm}^{f}$ is due to the doubly degenerate eigenvalues $\pm \frac{1}{2} \Omega_{+}$. Combining Eqs. (19) and (23), we find the energy eigenvalues $E$ of the super-Hamiltonian $H$,

$$
\begin{aligned}
E & =E_{b}-E_{f} \\
& =\left(n_{+}^{b}+\frac{1}{2} n_{+}^{f} n_{-}^{f}+\frac{1}{2}\right) \Omega_{+},
\end{aligned}
$$

where the quantum numbers $n_{ \pm}^{b}$ can take any non-negative integer values, while the 'fermionic' quantum numbers $n_{ \pm}^{f}$ can take only two values, either -1 or 1 . 
For $\Omega_{-}=0$, a reduction in the phase-space occurs. The phase-space variables $p_{2}$ and $x_{2}$ completely decouple from $H_{b}$. The energy eigenvalues $E_{b}$ of the bosonic sector and consequently $E$ becomes infinitely degenerate. Similarly, the four eigenvalues of $H_{f}$ reduces to two with each of them being doubly degenerate. The groundstate energy $E=0$ is

obtained, either for (i) $n_{+}^{b}=0, n_{+}^{f}=1, n_{-}^{f}=-1$ or $(\mathrm{ii}) n_{+}^{b}=0, n_{+}^{f}=-1, n_{-}^{f}=1$. The supersymmetry is unbroken.

The condition $\kappa=0$ has two solutions, (a) $\omega=0$ and (b) $1-B \theta+\phi_{1} \phi_{2}=0$. In the limit $\omega \rightarrow 0, \Omega_{+}=B$ and the eigenvalue equation of $H$ reduces to that of Pauli equation on the two dimensional commutative space. Note that the Pauli Hamiltonian in our case has higher symmetry than the one usually considered in the literature [27], since we have taken $4 \times 4$ dimensional matrix representation of the Clifford algebra instead of the familiar $2 \times 2$ dimensional representation. So, the degeneracy structure of the energy spectrum is different from Ref. 27].

\section{B. Non-critical phase $\kappa \neq 0$}

In the non-critical phase, both $\Omega_{ \pm} \neq 0$. The eigenvalue $E_{f}$

$$
-E_{f}=\frac{1}{2}\left(n_{-}^{f} \Omega_{-}+n_{+}^{f} \Omega_{+}\right)
$$

is identical to that of an anisotropic 'fermionic' oscillator with two degrees of freedom. The eigenvalue $E$ of the superHamiltonian $H$ is,

$$
E=\left(n_{+}^{b}+\frac{1}{2} n_{+}^{f}+\frac{1}{2}\right) \Omega_{+}+\left(n_{-}^{b}+\frac{1}{2} n_{-}^{f}+\frac{1}{2}\right) \Omega_{-} .
$$

The spectrum $E$ in Eq. (26) is identical to that of an anisotropic superoscillator on the commutative plane. The groundstate energy $E=0$ is obtained for $n_{ \pm}^{b}=0, n_{ \pm}^{f}=-1$. All excited states are paired together. The supersymmetry is unbroken.

The isotropic superoscillator can be obtained as a special case, when the parameters $B, \theta, \omega, \phi_{1,2}$ satisfy the following two relations simultaneously,

$$
\omega^{2} \theta+B=0, \quad \phi_{1}=\phi_{2}, \quad \kappa>0 .
$$


For $\kappa<0$, there is no 'isotropic limit'. The commutative limit, $B, \theta, \phi_{1,2} \rightarrow 0$ is of-course a solution of the above equation (27) for which $\Omega_{ \pm}=\omega^{2}$. Note that the isotropic superoscillator can be obtained as a special case only when both $\theta$ and $B$ are either zero or nonzero simultaneously.

\section{Inverted superoscillator on the noncommutative plane}

In the commutative space, the inverted harmonic oscillator

$$
\mathcal{H}=\frac{1}{2} \sum_{i=1}^{2}\left(p_{i}^{2}-\bar{\omega}^{2} x_{i}^{2}\right)
$$

with $\bar{\omega}$ real, is unbounded from below and there is no well defined groundstate. However, for the noncommutative case with both $B$ and $\theta$ being nonzero, $\bar{H}_{b}$ admits well defined groundstate 20]. This result can be understood intuitively as follows. The fundamental uncertainty relations are modified due to the non-commutativity and lower bounds appearing in these relations are expected to explicitly depend on $\theta$ and $B$. These parameters act as a kind of regulators/cut-offs to the singular potential for some ranges of their allowed values. Consequently, the potential effectively becomes bounded from below and admits well defined groundstate.

To analyze a similar situation in case of the supersymmetric inverted oscillator, we make the transformation $\omega \rightarrow i \bar{\omega}$ in all previous calculations in this section and identify any operator/function $A$ undergoing such transformation as $\bar{A}$. The transformation $\omega \rightarrow i \bar{\omega}$ amounts to taking a complex superpotential. The supercharges $\bar{Q}_{1}, \bar{Q}_{2}$ and the Hamiltonian $\bar{H}_{f}$ are no more hermitian operators. Further, unlike the standard supersymmetric theory, the supersymmetric Hamiltonian $\bar{H}$ is not a semi-positive definite quantity. These features are common to inverted superoscillator both on the commutative as well as on the noncommutative space. The inverted superoscillator on the commutative space do not have a well defined ground state. However, in spite of the undesirable features like non-hermiticity and unboundedness from below, we will show that the spectrum of the inverted superoscillator on the noncommutative space is indeed real. It is known that a certain class of 
non-hermitian Hamiltonian on the ordinary commutative space admits real spectra [28, 29]. To the best of our knowledge, the discussion in this section is the first example of a nonhermitian Hamiltonian admitting real spectra in the context of a supersymmetric theory as well as on a noncommutative space.

We find the frequencies of $\bar{H}_{b}$,

$$
\begin{aligned}
2 \bar{\Omega}_{ \pm} & =\sqrt{\left(\bar{\omega}^{2} \theta-B\right)^{2}-\bar{\omega}^{2}\left(\phi_{1}-\phi_{2}\right)^{2}+4 \bar{\kappa}} \pm \sqrt{\left(\bar{\omega}^{2} \theta-B\right)^{2}-\bar{\omega}^{2}\left(\phi_{1}-\phi_{2}\right)^{2}}, \bar{\kappa}>0, \\
2 \bar{\Omega}_{ \pm} & = \pm \sqrt{\left(\bar{\omega}^{2} \theta-B\right)^{2}-\bar{\omega}^{2}\left(\phi_{1}-\phi_{2}\right)^{2}+4 \bar{\kappa}}+\sqrt{\left(\bar{\omega}^{2} \theta-B\right)^{2}-\bar{\omega}^{2}\left(\phi_{1}-\phi_{2}\right)^{2}}, \bar{\kappa}<0, \\
\bar{\Omega}_{+} & \equiv \Omega=\sqrt{\left(\bar{\omega}^{2} \theta-B\right)^{2}-\bar{\omega}^{2}\left(\phi_{1}-\phi_{2}\right)^{2}}, \quad \Omega_{-}=0, \quad \bar{\kappa}=0,
\end{aligned}
$$

where $\bar{\kappa}=-\bar{\omega}^{2}\left(1-B \theta+\phi_{1} \phi_{2}\right)$. We discuss the three cases separately.

(i) $\bar{\kappa}<0$ : The commutative limit, $\left(\phi_{1,2}, \theta, B\right) \rightarrow 0$, can be taken only in the phase $\bar{\kappa}<0$. We find a complex $\bar{\Omega}_{ \pm}$in this limit, implying that the solutions are not stable. The commutative limit with nonzero magnetic field, i.e. $\left(\phi_{1,2}, \theta\right) \rightarrow 0 \& B \neq 0$, also belongs to this phase. The frequencies $\bar{\Omega}_{ \pm}$are complex for $B^{2}<4|\bar{\kappa}|$ and $\Omega_{-}$is negative for $B^{2}>4|\bar{\kappa}|$ signifying physically non-acceptable non-normalizable solutions. In-fact, in the phase $\bar{\kappa}<0$, there are no physically acceptable solutions, since $\bar{\Omega}_{ \pm}$are either complex or negative.

(ii) $\bar{\kappa}>0$ : There are regions in the parameter space for which physically acceptable solutions with well defined ground state energy can be found. In particular, for $\left(\bar{\omega}^{2} \theta-B\right)^{2} \geq \bar{\omega}^{2}\left(\phi_{1}-\right.$ $\left.\phi_{2}\right)^{2}$, both $\bar{\Omega}_{ \pm}$are real and positive. When the bound is saturated, we get an isotropic oscillator with $\bar{\Omega}_{+}=\bar{\Omega}_{-}=\sqrt{\bar{\kappa}}$. The energy spectrum is given by $\bar{E}=\left(n_{-}^{b}+\frac{1}{2} n_{-}^{f}+\frac{1}{2}\right) \bar{\Omega}_{-}+$ $\left(n_{+}^{b}+\frac{1}{2} n_{-}^{f}+\frac{1}{2}\right) \bar{\Omega}_{+}$with the zero groundstate energy.

(iii) $\bar{\kappa}=0$ : At the critical point $\bar{\kappa}=0$ there is a reduction in the phase space. For $\left(\bar{\omega}^{2} \theta-\right.$

$B)^{2}>\bar{\omega}^{2}\left(\phi_{1}-\phi_{2}\right)^{2}, \Omega$ is real and positive. The energy spectrum is, $\bar{E}=\left(n_{+}^{b}+\frac{1}{2} n_{+}^{f}+\frac{1}{2}\right) \Omega$ with a well defined ground state $\bar{E}_{0}=0$. The energy spectrum is infinitely degenerate.

\section{THREE DIMENSIONAL SUPEROSCILLATOR}

In this section, we discuss three dimensional superoscillator on the noncommutative space. For simplicity, we choose $C_{i j}=0 \forall i \& j$ so that the canonical commutation relations 
between the non-commutative coordinates and the momenta now read, $\left[\hat{x}_{i}, \hat{p}_{j}\right]=i \delta_{i j}$. Each of the antisymmetric matrices $B_{i j}$ and $\theta_{i j}$ is parameterized in terms of three parameters. In particular,

$$
B_{i j} \equiv \epsilon_{i j k} B_{k}, \quad \theta_{i j} \equiv \epsilon_{i j k} \theta_{k}
$$

We also define,

$$
B \equiv \sqrt{B_{1}^{2}+B_{2}^{2}+B_{3}^{2}}, \quad \theta \equiv \sqrt{\theta_{1}^{2}+\theta_{2}^{2}+\theta_{3}^{2}}
$$

for convenience. The bosonic part of the Hamiltonian is still given by $H_{b}$ in (14) with $N=3$. As is well known [15, 19] and described in Appendix A, the Hamiltonian $H_{b}$ can be expressed solely in terms of canonically conjugate variables $x_{i}$ and $p_{i}$ as an anisotropic oscillator in three dimensional commutative space. In particular,

$$
H_{b}=\frac{1}{2}\left[\Omega_{+}\left(p_{1}^{2}+x_{1}^{2}\right)+\Omega_{-}\left(p_{2}^{2}+x_{2}^{2}\right)+\Omega_{0}\left(p_{3}^{2}+x_{3}^{2}\right)\right]
$$

where $\pm \Omega_{ \pm}, \pm \Omega_{0}$ are the six eigenvalues of the $6 \times 6$ matrix $i M$ given in appendix A with $C_{i j}=0 \forall i \& j$. There are two phases of the theory characterized by $\kappa>0$ or $\kappa<0$ with $\kappa=0$ being the critical phase, where $\kappa \equiv \Omega_{+} \Omega_{-} \Omega_{0}$. The frequencies $\Omega_{ \pm}, \Omega_{0}$ are always positive for the range of parameters that is determined depending on the particular phase $(\kappa=0, \kappa>0, \kappa<0)$ in which the superoscillators are being considered.

The Hamiltonian $H_{f}$ with the parametrization (30) now reads,

$$
H_{f}=\frac{\omega}{2} \sum_{i=1}^{3} \xi_{i} \xi_{3+i}+\frac{i}{4} \sum_{i, j=1}^{3} \epsilon_{i j k}\left(B_{k} \xi_{i} \xi_{j}-\omega^{2} \theta_{k} \xi_{3+i} \xi_{3+j}\right) .
$$

We choose the matrix representation of the Clifford algebra (2),

$$
\begin{aligned}
& \xi_{1}=i \sigma_{3} \otimes \sigma_{2} \otimes I, \quad \xi_{2}=i \sigma_{1} \otimes \sigma_{2} \otimes I, \quad \xi_{3}=i \sigma_{2} \otimes I \otimes \sigma_{3}, \\
& \xi_{4}=\sigma_{2} \otimes I \otimes \sigma_{1}, \quad \xi_{5}=I \otimes \sigma_{3} \otimes \sigma_{2}, \quad \xi_{6}=I \otimes \sigma_{1} \otimes \sigma_{2} .
\end{aligned}
$$

The Hamiltonian $H_{f}$ in terms of these matrix representation of the elements of the Clifford algebra is given in Appendix B.

A general analysis of the spectrum of $H$ involves the diagonalization of the $6 \times 6$ matrix $M$ and $8 \times 8$ matrix $H_{f}$. The eigenvalues of both of these matrices come into positivenegative pairs. Thus, all the eigenvalues of $H$ can be obtained analytically by solving a 
cubic and a quartic characteristic equation, respectively. However, the final expressions for the frequencies $\Omega_{i}$ are quite messy and complicated. We present here results for a few specific choices of parameters with $\omega>0$. The results for $\omega<0$ can be obtained trivially by considering the negative pairs of the frequencies $\Omega_{ \pm}, \Omega_{0}$. The results for the purely bosonic model has been obtained previously for some of the cases considered below, while the results for the fermionic part and hence, the complete supersymmetric model are obtained for the first time in this paper. We would like to mention here that the supersymmetry is unbroken for all the cases considered below.

(a) $\mathbf{B}_{\mathbf{i}}=\mathbf{0}, \forall \mathbf{i}$ : The noncommutativity among the momentum operators are switched off. There is only one phase characterized by $\kappa=\omega^{3}>0$ for positive $\omega$. The frequencies are,

$$
\Omega_{0}=\omega, \quad \Omega_{ \pm}=\omega \sqrt{t \pm \sqrt{t^{2}-1}}, \quad t \equiv 1+\frac{1}{2} \omega^{2} \theta^{2}
$$

There is no limit for nonzero $\theta_{i}$ 's for which $\Omega_{+}=\Omega_{-}$. The eight eigenvalues of $H_{f}$,

$$
\left\{\frac{\omega}{2}(1 \pm \sqrt{2+2 t}), \quad-\frac{\omega}{2}(1 \pm \sqrt{2+2 t}), \quad \frac{\omega}{2}(1 \pm \sqrt{2 t-1}), \quad-\frac{\omega}{2}(1 \pm \sqrt{2 t-1})\right\}
$$

can be written in a closed form through the introduction of three quantum numbers $n_{ \pm}^{f}, n_{0}^{f}$,

$$
-E_{f}=\frac{1}{2}\left(n_{-}^{f} \Omega_{-}+n_{+}^{f} \Omega_{+}+n_{0}^{f} \Omega_{0}\right), \quad n_{ \pm}^{f}, n_{0}^{f}=-1,1
$$

Thus, the eigenspectrum of $H$,

$$
E=\left(n_{+}^{b}+\frac{1}{2} n_{+}^{f}+\frac{1}{2}\right) \Omega_{+}+\left(n_{-}^{b}+\frac{1}{2} n_{-}^{f}+\frac{1}{2}\right) \Omega_{-}+\left(n_{0}^{b}+\frac{1}{2} n_{0}^{f}+\frac{1}{2}\right) \Omega_{0},
$$

is that of an anisotropic superoscillator on the three dimensional commutative space. The bosonic quantum numbers $n_{ \pm}^{b}, n_{0}^{b}$ can take any non-negative integer values. The supersymmetric groundstate $E=0$ is obtained for $n_{ \pm}^{b}=n_{0}^{b}=0, n_{ \pm}^{f}=n_{0}^{f}=-1$.

(b) $\mathbf{B}_{\mathbf{i}}=-\omega^{2} \theta_{\mathbf{i}}, \forall \mathbf{i}$ : For this choice, two of the three frequencies become equal,

$$
\Omega_{0}=\omega, \quad \Omega_{+}=\Omega_{-} \equiv \Omega=\omega \sqrt{1+\omega^{2} \theta^{2}}
$$

producing an isotropic oscillator on the $\mathrm{x}-\mathrm{y}$ plane plus an oscillator along the z-direction. There is again only one phase $\kappa>0$ for positive $\omega$. The eight eigenvalues of $H_{f}$ can 
be expressed in a closed form as in (37) with the frequencies given by Eq. (39). The eigenspectrum of $H$,

$$
E=\left(n_{+}^{b}+n_{-}^{b}+\frac{1}{2} n_{+}^{f}+\frac{1}{2} n_{-}^{f}+1\right) \Omega+\left(n_{0}^{b}+\frac{1}{2} n_{0}^{f}+\frac{1}{2}\right) \Omega_{0},
$$

has higher level of degeneracy compared to (38). The supersymmetric groundstate is obtained for all bosonic quantum numbers being zero and all the fermionic quantum numbers equal to -1 .

(c) $\mathbf{B}_{\mathbf{i}}=\omega^{\mathbf{2}} \theta_{\mathbf{i}}, \forall \mathbf{i}$ : There are two phases in the theory characterized by $\kappa=\omega^{3}\left(1-\omega^{2} \theta^{2}\right)$. The frequencies in these two phases are,

$$
\begin{aligned}
& \Omega_{0}=\omega, \quad \Omega_{ \pm}=\omega(1 \pm \omega \theta), \quad \kappa>0, \\
& \Omega_{0}=\omega, \quad \Omega_{ \pm}=\omega( \pm 1+\omega \theta), \quad \kappa<0 .
\end{aligned}
$$

There is no isotropic point $\Omega_{-}=\Omega_{+}$for $\theta_{i} \neq 0$. The eigenspectrum of $H$ is given by Eq. (38) with the frequencies are as in Eq. (41).

(d) Critical point $\kappa=0$ : The critical point $\kappa=0$ separating the two phases of the superoscillators is determined from the relation, $\operatorname{Det}(M)=\omega^{6}\left(1-\sum_{i=1}^{3} \theta_{i} B_{i}\right)=0$. There are two cases, (i) $\sum_{i=1}^{3} \theta_{i} B_{i}=1$ and (ii) $\omega=0$.

(i) $\sum_{i=1}^{3} \theta_{i} B_{i}=1$ : Without any loss of generality, we choose a particular solution, $B_{i}=$ $\theta_{i} \theta^{-2}$. The phase-space is reduced to 4 dimensions, since one of the frequency vanishes. In particular,

$$
\Omega_{0}=0, \quad \Omega_{+}=\omega, \quad \Omega_{-}=\theta^{-1}\left(1+\omega^{2} \theta^{2}\right)
$$

The bosonic spectrum becomes infinitely degenerate. At the critical point, there are four independent eigenvalues of $H_{f}$ with each of them being doubly degenerate,

$$
\left\{ \pm \frac{\omega}{2}+\frac{1}{2 \theta}\left(1+\theta^{2} \omega^{2}\right), \pm \frac{\omega}{2}-\frac{1}{2 \theta}\left(1+\theta^{2} \omega^{2}\right)\right\}
$$

The eigenvalues of $H$ can be written in a closed form as,

$$
E=\left(n_{+}^{b}+\frac{1}{2} n_{0}^{f} n_{+}^{f}+\frac{1}{2}\right) \Omega_{+}+\left(n_{-}^{b}+\frac{1}{2} n_{0}^{f} n_{-}^{f}+\frac{1}{2}\right) \Omega_{-} .
$$


The $E=0$ groundstate is obtained for (i) $n_{+}^{b}=0, n_{0}^{f}=1, n_{ \pm}^{f}=-1$ and (ii) $n_{+}^{b}=0, n_{0}^{f}=$ $-1, n_{ \pm}^{f}=1$. The whole spectrum including the groundstate is of-course infinitely degenerate due to the reduction in the phase-space.

(ii) $\omega=0$ : The eigenvalue equation of the Hamiltonian $H$ is that of a three dimensional Pauli equation. There are further reductions in the phase space, compared to the case described above. Four out of the six phase space variables decouple from the dynamics. In particular,

$$
\Omega_{ \pm}=0, \quad \Omega_{0}=B
$$

The eigenvalues of $H_{f}$ are $\pm \frac{1}{2} \Omega_{0}$ with each of them having four-fold degeneracy. The eigenspectrum of $H$ can be written as,

$$
E=\left(n_{0}^{b}+\frac{1}{2} n_{0}^{f} n_{-}^{f} n_{+}^{f}+\frac{1}{2}\right) \Omega_{0} .
$$

The $E=0$ groundstate can be obtained for (i) $n_{0}^{b}=0, n_{0}^{f}=-1, n_{ \pm}^{f}=1$, (ii) $n_{0}^{b}=0, n_{0}^{f}=$ $-1, n_{ \pm}^{f}=-1$, (iii) $n_{0}^{b}=0, n_{0}^{f}=1, n_{ \pm}^{f}= \pm 1$ and (iv) $n_{0}^{b}=0, n_{0}^{f}=1, n_{ \pm}^{f}=\mp 1$. The whole spectrum including the groundstate is of-course infinitely degenerate due to the reduction in the phase-space.

\section{SUMMARY \& DISCUSSIONS}

We have constructed supersymmetric quantum mechanics on the noncommutative space in terms of $\mathcal{N}=2$ real supercharges. This construction is valid in any arbitrary dimensions for arbitrary superpotential. Further, the same construction is valid for a very large class of noncommutativities among phase-space operators. The non-commutativity among the space coordinates and/or among momentum coordinates restricts the number of independent ways one can construct $\mathcal{N}=2$ supersymmetric theory for a given superpotential.

We have studied the noncommutative superoscillators in two and three dimensions in some detail and obtained their eigenspectra analytically. The spectrum of the noncommutative superoscillator in two(three) dimensions is identical to an anisotropic oscillator in

the commutative two(three) space dimensions. There is a critical surface in the parameter 
space for which there is reduction in the phase-space. Consequently, the spectrum due to the bosonic sector become infinitely degenerate, while the degeneracy in the fermionic sector is doubled. This is because the bosonic quantum numbers can take any non-negative integer values, while the fermionic quantum numbers can take only two values.

We have studied the 'inverted superoscillator' on the noncommutative plane. The corresponding Hamiltonian is neither hermitian nor a semi-positive definite operator. Inspite of these, we have found the spectrum to be real, positive and with a well defined ground state for a few specific choices of the parameters. For these choices of parameters, there is no smooth limit to the ordinary commutative space. Thus, the energy spectrum with a well defined ground state for an 'inverted superoscillator' is purely the effect of noncommutativity.

\section{Acknowledgments}

It is a great pleasure to acknowledge several useful discussions with Prof. Avinash Khare. I also thank him for a very critical reading of the manuscript. This work is supported (DO No. SR/FTP/PS-06/2001) by SERC, DST, Govt. of India through the Fast Track Scheme for Young Scientists:2001-2002.

\section{APPENDIX A: MAPPING OF $H_{b}$ TO AN ANISOTROPIC OSCILLATOR ON COMMUTATIVE SPACE FOR $N=3$}

In this Appendix, we describe a general procedure for mapping the non-commutative Hamiltonian $H_{b}$ to an equivalent Hamiltonian of anisotropic oscillators on the commutative space. The discussion is based on Refs. [15, 19, 30].

Define a 6 dimensional vector $U=\left(\omega \hat{x}_{1}, \hat{p}_{1}, \omega \hat{x}_{2}, \hat{p}_{2}, \omega \hat{x}_{3}, \hat{p}_{3}\right)$ in the phase space. The algebra in (11) can be written as,

$$
\left[U_{I}, U_{J}\right]=i M_{I J}, \quad I, J=1,2, \ldots 6
$$


where the $6 \times 6$ dimensional matrix $M$ is given by,

$$
M=\left(\begin{array}{cccccc}
0 & \omega & \omega^{2} \theta_{3} & \omega C_{12} & -\omega^{2} \theta_{2} & \omega C_{13} \\
-\omega & 0 & -\omega C_{21} & B_{3} & -\omega C_{31} & -B_{2} \\
-\omega^{2} \theta_{3} & \omega C_{21} & 0 & \omega & \omega^{2} \theta_{1} & \omega C_{23} \\
-\omega C_{12} & -B_{3} & -\omega & 0 & -\omega C_{32} & B_{1} \\
\omega^{2} \theta_{2} & \omega C_{31} & -\omega^{2} \theta_{1} & \omega C_{32} & 0 & \omega \\
-\omega C_{13} & B_{2} & -\omega C_{23} & -B_{1} & -\omega & 0
\end{array}\right) .
$$

There exists an orthogonal transformation such that the matrix $M$ can be block-diagonalized $\operatorname{as}[30]$,

$$
R^{T} M R=\left(\begin{array}{ccc}
D_{1} & 0 & \\
0 & D_{2} & 0 \\
0 & 0 & D_{3}
\end{array}\right), \quad D_{i} \equiv\left(\begin{array}{cc}
0 & \Omega_{i} \\
-\Omega_{i} & 0
\end{array}\right),
$$

using a $O(6)$ rotational matrix $R$ and its transpose $R^{T}$, where $\pm \Omega_{i}$ are the six eigenvalues of $i M$. The matrix $R$ is unique modulo $O(6)$ rotations. The transformed variables in the phase space, $\left(x_{1}, p_{1}, x_{2}, p_{2}, x_{3}, p_{3}\right) \equiv\left(\frac{u_{1}}{\sqrt{\left|\Omega_{1}\right|}}, \frac{u_{2}}{\sqrt{\left|\Omega_{1}\right|}} \frac{u_{3}}{\sqrt{\left|\Omega_{2}\right|}} \frac{u_{4}}{\sqrt{\left|\Omega_{2}\right|}} \frac{u_{5}}{\sqrt{\left|\Omega_{3}\right|}} \frac{u_{6}}{\sqrt{\left|\Omega_{3}\right|}}\right)$ with $u=R^{T} U$ satisfy the usual canonical commutation relations

$$
\left[x_{i}, x_{j}\right]=0, \quad\left[p_{i}, p_{j}\right]=0, \quad\left[x_{i}, p_{j}\right]=i \delta_{i j}
$$

The Hamiltonian $H_{b}=\frac{1}{2} \sum_{I=1}^{6} U_{I}^{2}=\frac{1}{2} \sum_{I=1}^{6} u_{I}^{2}$, because of the $O(6)$ invariance. We get back the Hamiltonian (32) once we express the variables $u_{I}$ in terms of $x_{i}$ and $p_{i}$.

For $N=2$, We can truncate the matrix $M$ to a $4 \times 4$ matrix by taking the first four row and columns only, i.e. $M_{I J}$ with $I, J=1,2,3,4$. Identifying $\theta_{3}=\theta, B_{3}=B, C_{12}=\phi_{1}$ and $c_{21}=-\phi_{2}$, all the relevant results of Sec. III can be obtained.

\section{APPENDIX B: $H_{f}$ IN MATRIX REPRESENTATION FOR $N=3$}

The Hamiltonian $H_{f}$ in terms of the matrix representation of the Clifford algebra has the following form,

$$
H_{f}=\left(\begin{array}{cc}
\Gamma_{1} & \eta \\
\eta^{\dagger} & \Gamma_{2}
\end{array}\right),
$$


where the $4 \times 4$ matrices $\Gamma_{1}, \Gamma_{2}$ and $\eta$ are,

$$
\begin{aligned}
& \Gamma_{1} \equiv\left(\begin{array}{cccc}
0 & -\gamma_{3}^{+} & -\gamma_{1}^{+} & -\gamma_{2}^{-} \\
\gamma_{3}^{+} & 0 & -\gamma_{2}^{+} & \gamma_{1}^{-} \\
\gamma_{1}^{+} & \gamma_{2}^{+} & 0 & -\gamma_{3}^{-} \\
\gamma_{2}^{-} & -\gamma_{1}^{-} & \gamma_{3}^{-} & 0
\end{array}\right), \Gamma_{2} \equiv\left(\begin{array}{cccc}
0 & -\gamma_{3}^{-} & \gamma_{1}^{-} & \gamma_{2}^{-} \\
\gamma_{3}^{-} & 0 & \gamma_{2}^{+} & -\gamma_{1}^{+} \\
& & & \\
-\gamma_{1}^{-} & -\gamma_{2}^{+} & 0 & -\gamma_{3}^{+} \\
& & & \\
-\gamma_{2}^{-} & \gamma_{1}^{+} & \gamma_{3}^{+} & 0
\end{array}\right) \text {, } \\
& \eta \equiv\left(\begin{array}{cccc}
0 & 0 & 0 & -\frac{i}{2} \omega \\
0 & 0 & \frac{i}{2} \omega & 0 \\
0 & \frac{i}{2} \omega & 0 & 0 \\
\frac{3 i}{2} \omega & 0 & 0 & 0
\end{array}\right), \quad \gamma_{i}^{ \pm} \equiv \frac{i}{2}\left(B_{i} \pm \theta_{i} \omega^{2}\right)
\end{aligned}
$$

Note that,

$$
\left(\gamma_{i}^{ \pm}\right)^{*}=-\gamma_{i}^{ \pm}
$$

where $(X)^{*}$ denotes the complex conjugate of $X$. The hermiticity of $H_{f}$ can now be checked easily.

[1] A. Connes, Noncommutative zeometry, Academic Press, London, (1994).

[2] N. Seiberg and E. Witten, JHEP 9909 (1999) 032 hep-th/9908142.

[3] S. Minwalla, M. V. Raamsdonk, N. Seiberg, JHEP 0002 (2000) 020 hep-th/9912072. 
[4] R. Gopakumar, S. Minwalla and A. Strominger, JHEP 0005 (2000) 020 hep-th/0003160.

[5] M. R. Douglas and N. A. Nekrasov, Rev. Mod. Phys. 73 (2001) 977 hep-th/0106048.

[6] J. A. Harvey, Komaba Lectures on Noncommutative Solitons and D-Branes, hep-th/0102076.

[7] R. J. Szabo, Int. J. Mod. Phys. A19 (2004) 1837 physics/0401142; Phys. Rep. 378 (2003) 207 hep-th/0109162.

[8] V. O. Rivelles, hep-th/0305122.

[9] D. Klemm, S. Penati and L. Tamassia, Class. Quant. Grav. 20 (2003) 2905 hep-th/0104190.

[10] N. Seiberg, JHEP 0306 (2003) 010 hep-th/0305248.

[11] C. Duval and P. Horvathy, Phys. Lett. B479 (2000) 284 hep-th/0002233; J. Phys. A34 (2001) 10097 hep-th/0106089; P. Horvathy, Ann. Phys. 299 (2002) 128 hep-th/0201007.

[12] M. Chaichian, M. M. Sheikh-Jabbari and A. Tureanu, Phys. Rev. Lett. 86 (2001) 2716 hep-th/0010175.

[13] M. Chaichian, P. Presnajder, M. M. Sheikh-jabbari and A. Tureanu, Phys. Lett. B527 (2002) hep-th/0012175.

[14] V. P. Nair and A. P. Polychronakos, Phys. Lett. B505 (2001) 267 hep-th/0011172.

[15] A. Hatzinikitas and I. Smyrnakis, J. Math. Phys. 43 (2002) 113 hep-th/0103074.

[16] A. Jellal, J. Phys. A34 (2001) 10159 hep-th/0105303; S. Bellucci, A. Nersessian and C. Sochichiu, Phys. Lett. B52 (2001) 345 hep-th/0106138; A. Smailagic and E. Spalluci, Phys. Rev. D65 (2002) 107701 hep-th/0108216; B. Muthukumar and P. Mitra, Phys. Rev. D66 (2002) 027701 hep-th/0204149.

[17] R. Banerjee, Mod. Phys. Lett. A17 (2002) 631 [0106280].

[18] A. Smailagic and E. Spallucci, J. Phys. A365 (2002) L363 hep-th/0205242.

[19] L. Jonke and S. Meljanac, Eur. Phys. J. C29 (2003) 433 hep-th/0210042; I. Dadic, L. Jonke and S. Meljanac, hep-th/0301066

[20] S. Bellucci, Phys. Rev D67 (2003) 105014 hep-th/0301227.

[21] F. Cooper, A. Khare and U. Sukhatme, Phys. Rep. 251 (1995) 267.

[22] T. Curtright, D. Fairlie and C. K. Zachos, Phys. Rev. D58 (1998) 025002 hep-th/9711183. 
[23] E. Harikumar, V. Sunil Kumar and A. Khare, Phys. Lett. B589 (2004) 155 hep-th/0402064.

[24] P. Pouliot, Class. Quant. Grav. 21 (2004) 145 hep-th/0306261.

[25] P. K. Ghosh, Nucl. Phys. B681 (2004) 359 hep-th/0309183.

[26] E. D'Hoker and L. Vinet, Comm. Math. Phys. 97 (1985) 391.

[27] M. de Crombrugghe and V. Rittenberg, Ann. Phys. 151 (1983) 99; A. Khare and J. Maharana, Nucl. Phys. B244 (1984) 409.

[28] C. M. Bender, D. C. Brody and H. F. Jones, Am. J. Phys. 71 (2003) 1095 hep-th/0303005; C.M. Bender and S. Boettcher, Phys. Rev. Lett. 80 (1998) 5243.

[29] P. K. Ghosh, quant-ph/0501087

[30] D. McDuff and D. Salamon, Introduction to Symplectic Topology, Oxford Science Publications (1998). 